\title{
Surrounding Space On the Ontology of Organism-Environment Relations
}

\author{
Barry Smith \\ Department of Philosophy and Center for Cognitive Science, SUNY Buffalo, NY
}

Achille C. Varzi

Department of Philosophy, Columbia University, New York, NY

[Published in Theory in Biosciences 120:2 (2002), 139-162]

\begin{abstract}
The history of evolution is a history of development from less to more complex organisms. This growth in complexity of organisms goes hand in hand with a concurrent growth in complexity of environments and of organism-environment relations. It is a concern with this latter aspect of evolutionary development that motivates the present paper. We begin by outlining a theory of organism-environment relations. We then show that the theory can be applied to a range of different sorts of cases, both biological and non-biological, in which objects are lodged or housed within specific environments, or niches. Biological science is interested in types - for example in genotypes, phenotypes, and environment types - in regularities that can serve as the basis for the formulation of laws or general principles. Types, however, can exist only through their corresponding tokens. Our theory of token environments is meant to plug this gap and to provide a first step towards a general theory of causally relevant spatial volumes.
\end{abstract}

\section{Introduction}

The history of evolution is, very roughly, a history of development from the less to the more complex. Philosophers who have turned their attentions to these matters have concentrated almost exclusively on the growth in complexity of organisms. (In this, of course, they have followed most biologists.) As Richard Lewontin [21] points out, however, the growth in complexity of organisms goes hand in hand with a concurrent growth in complexity of environments and thus of organism-environment relations. Organisms select their environments, but they also determine and modify the environmental features which are most relevant to their own survival. It is this latter aspect of evolutionary development that concerns us here.

Consider the armchair in which you are now sitting. This is an artefact; an object of a special sort which is to be understood, on standard views, in 
terms of its function or in terms of the intentions of its creator. Yet there is an aspect of your armchair which it shares with non-artefactual entities such as the hollow in the ground in which an ape might shelter from the wind, or the cave in which a bear might make its home. Briefly, the armchair, the hollow, and the cave are all what we shall call niches, they are environments into which an organism fits. ${ }^{1}$ This fitting relation is pervasively exemplified not only in the realm of biological phenomena more narrowly conceived, but also in the realms of technology and culture. Indeed artefacts in general can themselves be divided into two sorts: those which are designed to serve in the fitting relation such as armchairs, rooms, parks, circus-tents; and those which are not so designed. Even the latter are in many cases standardly applied to the construction or embellishment of artefacts of the former type. This is true, for example, of carpenters' tools and of paintings or lampstands.

In [31], drawing primarily on biological examples, we defended a view of the relation of fit between a niche and its tenants as a relation capable of being specified with the aid of basic concepts of formal ontology-of mereology, topology, and the theory of location. The theory there presented was synchronic only. That is to say, we were concerned exclusively with the formal structure exemplified whenever a tenant is in a niche at a given time. In such circumstances a niche is analogous to a topological neighborhood surrounding its tenant. In the present paper we extend this theory by providing a first step towards a theory of niche dynamics, a theory of life, motion, and growth, via a more detailed account of the internal structures of niches and of the causal relations between niches and their surroundings. By adding this dynamic dimension we shall be in a position to throw light on the ways in which evolution involves simultaneous and mutually reinforcing processes of natural selection and niche construction.

Intuitively, a niche may or may not be occupied by a tenant. The tenant may leave its niche, as when the kangaroo joey leaves the pouch of its mother. Or the tenant may move from one niche to another, as when the whooping crane migrates from its breeding grounds in Alberta to its winter

\footnotetext{
${ }^{1}$ Dictionaries distinguish two senses of the term 'niche': an architectural sense, which means a crevice or hole, a place into which something fits, and a functional sense, which means a role or option within a space of alternatives. The relation between these uses of the term from an ecological perspective will be addressed below.
} 
home in the Gulf of Mexico. A theory of niche dynamics must therefore make room not only for a theory of the niche as occupied (i.e., of the tenantniche relation), but also for a theory of the vacant niche and of the passage from one niche to another. We thus begin our remarks with an account of what vacant and occupied niches have in common. As in the earlier paper, we shall concentrate primarily on biological examples. The niche structures with which we deal will in the first place be those that are instantiated where organisms or groups of organisms are housed within their surroundings, whether natural (the hollow or cave) or artefactual (the armchair, a house, a circus tent). Later, however, we will show that the theory of such structures can be applied to a wide range of different sorts of cases in which objects, including inanimate objects, are lodged or housed within environmental settings.

\section{The Structure of Niches}

Consider an occupied niche-the bear in its cave (Figure 1). There is manifested here what we might think of as a double hole structure. In the center of this structure is the bear itself, which, by displacing air, at one and the same time creates and occupies a central hole in its niche-a hole that is precisely the right size and shape to be occupied by this very bear. As the bear moves, the hole moves too- the hole is dependent for its existence on the bear and on the bear's being in this precise location. The two are quite literally inseparable. The bear is, in this regard, like a fish in the ocean: it is a perfect filler of a cavity in the interior of the surrounding medium-air in one case; water in the other. ${ }^{2}$ It is the medium that allows the bear to move and breathe. Surrounding and supporting this medium is an enclosing structure, or what we shall call a retainer, which in the present case is constituted by the walls and floor of the cave. We can accordingly think of the medium as filling a second, larger hole-an environing hole that is exactly as large as the interior of the cave, minus the bear. The environing hole is bounded by the retainer, and it surrounds the bear, and hence also it surrounds the central hole.

\footnotetext{
${ }^{2}$ Compare the section on making filled holes in [2], ch. 10.
} 


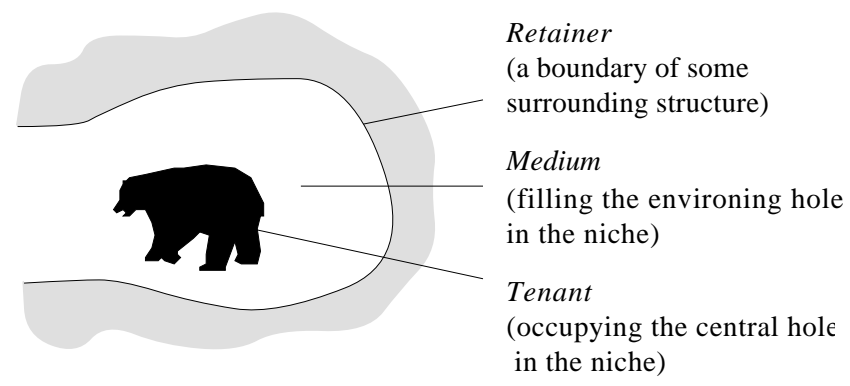

Figure 1. The double hole structure of the occupied niche.

A typical niche, we submit, exhibits a double hole structure of this sort. It is a bounded volume of space; it is the specific habitat, location, or site that is actually occupied by a given organism or group of organisms on a given occasion. ${ }^{3}$ It is an environment that is suitable or adequate for given purposes (foraging, resting, hunting, grooming) of an organism or group of organisms. This is close to David Lack's definition of ecological niches as: "the places where a species feeds within its habitat" [18]. Thus understood, a niche typically involves a medium as well as some retainer; for the retainer helps, by channeling causal forces, to keep the medium in its place. ${ }^{4}$

More standardly, however, the term 'niche' is used by ecologists to refer not to concrete spatial volumes but, rather, to something abstract which we can think of in terms of a job description, a functional role, or a resource utilization spectrum. ${ }^{5}$ A niche thus abstractly understood is a constellation of environmental variables - such as degree of slope, exposure to sunlight, soil fertility, foliage density, proximity and type of predators, and so on-which are relevant to the survival of organisms of a given species. In the terminology of G. E. Hutchinson [14], a niche is an abstract hypervolume in a many-

\footnotetext{
${ }^{3}$ Although our examples will standardly focus on niches whose tenants are individual organisms, a niche tenant need not be a single, connected individual. A family of bears in a cave is an aggregative tenant which includes separate organisms as proper parts. Some tenants of this sort are reproductively isolated subpopulations of conspecifics (or "avatars") that appear to play an important role in evolutionary theory $[4,5]$.

${ }^{4}$ Below we also consider the case of niches which lack a physical retainer.

${ }^{5}$ On the development of the ecological concept of niche, and the many senses of 'niche' in the ecological literature, see $[13,23]$.
} 
dimensional space determined by the relevant environmental properties. Every point in the interior of such a hypervolume corresponds to a state of the environment which permits the corresponding species to exist indefinitely. As an example, Figure 2 illustrates a classical Hutchinsonian niche for squirrels: the $x$ axis in the diagram defines food size (e.g., the mean diameter of acorns and other seeds or fruits); the $y$ axis defines temperature tolerance; and the $z$ axis defines 'some measure of the density of branches, between certain diameters, in unit volume of physical space'. Lewontin has a similar abstract notion in mind when he writes that "the use of the metaphor of a niche implies a kind of ecological space with holes in it that are filled by organisms, organisms whose properties give them the right "shape" to fit into the holes.' [22: 44] The holes, in this picture, are not spatial volumes within a surrounding medium but abstract loci within an idealized space of theoretical parameters.

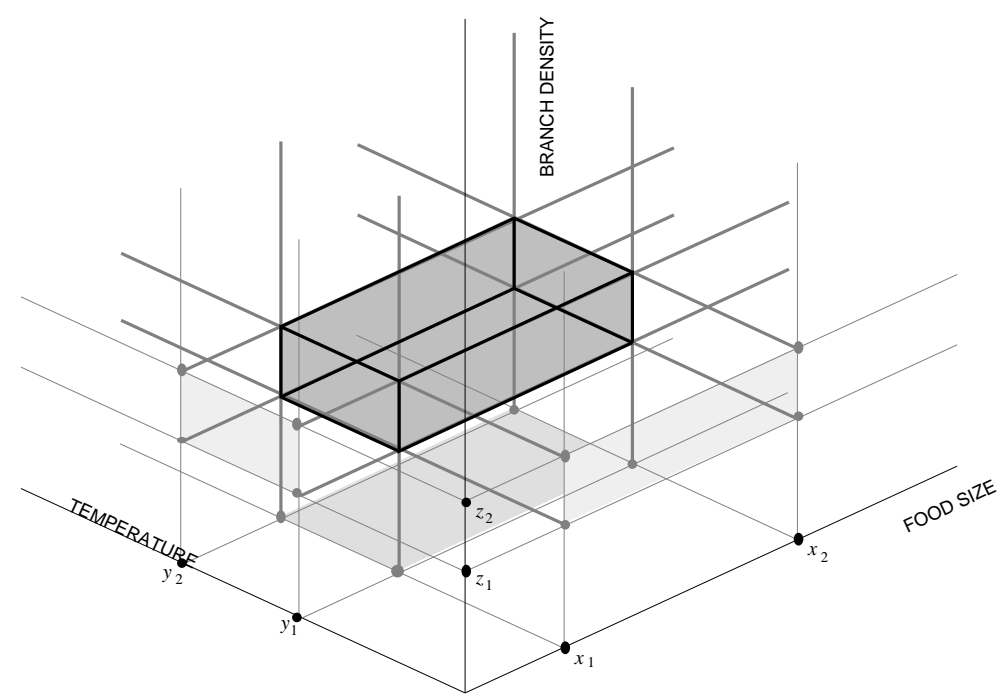

Figure 2. A three-dimensional ecological niche (after [14: 159]).

Clearly, these two notions of niche-as a concrete spatial volume and as an abstract hypervolume-must be in some way combined, since constellations of environmental variables are relevant to the survival of organisms 
only insofar as the corresponding properties are realized or instantiated at specific times in specific regions of space. This is recognized by E. P. Odum, for whom the niche of an organism includes not only 'its functional role in the community (as, for example, its trophic position) and its position in environmental gradients of temperature, moisture, $\mathrm{pH}$, soil and other conditions of existence' [25: 234], but also the physical space which the organism occupies.

Hutchinson himself distinguishes between the 'fundamental' niche (defined as the total hypervolume satisfying the conditions under which a species could live and replace itself) and the 'realized' niche (the portion of that hypervolume which is actually suited for that species at a given time, for example because competition excludes it from other portions). However, this distinction does not capture the opposition mentioned by Odum. Both fundamental and realized niches are volumes in an abstract space of environmental parameters. The physical space occupied by an organism, by contrast, sustains an actual instantiation of the realized niche of its species in some given physical location. ${ }^{6}$

In our [31] we sought to make sense of the relations involved here by introducing the notion of a spatialized niche as a tokenizing of some ecological niche type. The present paper offers a more detailed account of this conception. We can now assert that it is the medium of the niche in our sense which is at any given time the carrier of the various properties represented in the Hutchinsonian niche type. The medium of a concrete niche is what results when a given spatial region (of given altitude, orientation, exposure, etc.) comprehends air or water molecules (or some other stuff) instantiating environmental properties that fall within the relevant threshold values. Thus the medium of the bear's niche is constituted, in first approximation, by a body of air surrounding the bear, a body of air whose temperature, pressure, viscosity, etc. fall within the threshold values that define the ecological niche type for that sort of organism. And the retainer helps to ensure that the me-

\footnotetext{
${ }^{6}$ It would be more appropriate to say that the physical space occupied by an organism sustains an instantiation of a relevant portion of the realized niche of its species. For normally such a space sustains only some subset of the relevant environmental variables. (The bear's cave, for example, does not include those parts of the environment where the bear forages.) In the following, we shall take such qualifications for granted.
} 
dium satisfies these properties by setting it apart from its surroundings (though the retainer itself does not satisfy those properties). The medium of the niche of a particular squirrel on a particular occasion is likewise that body of air within which the squirrel breathes, eats, and moves-a body of air that occupies a region where temperature, branch density, and food size satisfy the conditions that define the Hutchinsonian hypervolume for that type of squirrel. The retainer, in this case, is a much more heterogeneous physical structure corresponding to the surfaces of the ground and trees that demarcate the relevant spatial region. But to the extent that such a structure supports the medium - to the extent that it helps the medium stay within the threshold values for the relevant Hutchinsonian parameters - to that extent it is part of the niche.

\section{The Medium for Life}

The medium of a niche is that into which the tenant fits-it is that which occupies the environing hole of the niche. Typically it consists of air, or water, or gases and liquids in general. (Water-in contrast to air-may in some circumstances serve as medium and in other circumstances serve as partial retainer, for example as the horizontal underlying support for the niches of small flies above the surface of a lake.) Rarely, however, will the medium of a niche be constituted in homogeneous fashion out of a single stuff. Rather, a niche will standardly involve a mixed medium, sometimes a hugely diverse combination of air plus water plus other nutrients and impurities, including radioactive impurities, as well as vitamins, amino acids, amniotic fluids, salts, and sugars in which organisms of different sorts, from protozoa to large mammals, may live.

The niche of a bear or of a squirrel, too, will standardly involve a medium consisting of air as well as of any nutrient (nuts, seeds, etc.) that fills the relevant environing hole. But the medium of a niche need not fill the relevant environing hole completely. For example, a bat flying in the cave in which the bear lives would occupy part of the environing hole of the bear's niche; but it would not be part of the pertinent medium. The surface of the bat would not be part of the retainer either, though it is part of the medium's boundary. In general, a physical surface is part of the relevant niche-retainer only if it is relevant to the behavioral and survival patterns of the tenant (in this case: the bear). 
Thus, not only are media necessary ingredients of niches. As the concept is here understood, a medium exists only within the context of a niche. Indeed, a body of stuff constitutes a medium in our technical sense only relative to a given type of niche, and thus only relative to a given type of organism. It is the type of organism that fixes the relevant hypervolume of environmental properties which the medium exemplifies. Accordingly, a medium requires a tenant or tenants of the relevant type in order to exist. In the case of an occupied niche, such as the cave of the bear, the medium surrounds the tenant, allowing the latter a degree of free play to grow and move. In the case of a vacant niche tenants of the relevant type must exist at least within accessible distance, the sense of "accessible" being determined in each case by the type of organism involved. In addition, a vacant niche requires a retainer by which the relevant medium is circumscribed and held in place. ${ }^{7}$ In this respect too, therefore, our account of the medium-retainer structure goes beyond the theory of niches in terms of formal concepts derived from mereology, topology, and the theory of location given in [31]. The organism-medium-retainer structure adds a material dimension.

Consider, now, what happens when a tenant leaves its niche. It seems to be a characteristic feature of such cases that the gap left by the tenant, or by any other object that is removed from the niche, is filled immediately by the surrounding medium. Modulo the elasticity of the retainer (consider the kangaroo joey leaving the pouch of its mother), this is an operational test for being a niche medium. If Luigi is buried in a hole filled with concrete, the latter is not a medium, because it does not pass our operational test. Luigi, accordingly, is not in a niche. If, as we are arguing, every niche has a medium of the appropriate sort, then there are for this reason no niches in cases where inanimate bodies of dense matter are housed within immediately surrounding bodies of equally dense matter (as Michelangelo's David, for example, was once housed inside a solid block of stone). ${ }^{8}$

\footnotetext{
${ }^{7}$ Thus, a medium is normally of lower physical density than both organism and retainer. It seems indeed reasonable to suppose that life can evolve, and can be sustained, only within a medium that is less dense than the organisms which live within it.

${ }^{8}$ On the other hand, the operational test seems to be complied with in the case of a valve in a piston, or a jewel in a jewel box. In such cases we would seem to be able to apply the basic niche concept even though the pertinent tenant is not alive.
} 
Does the operational test imply that every medium must to some extent be a mixed medium? Consider the body of air and gold coins in which Scrooge likes to immerse himself when celebrating his riches. Or consider what happens when a worm moves through a body of earth: a mixed medium then fills out the gaps created through this burrowing action, a medium that is constituted out of particles of earth and air. These examples draw attention to a feature possessed already by air and water themselves, namely, that they are manifested always in the form of air and water molecules. By our operational test every medium is maximal: it expands into every accessible nook and cranny of the relevant niche boundary. What, then, does the job of filling out the niche whose medium is made primarily of air or water? In the Scrooge and worm cases this job is performed by air. But what does the job in the case of air itself, conceived as a body of molecules separated by gaps?

Our answer to this question is that intervening bodies of empty space are involved wherever air (and water) molecules are gathered together to form a whole. It is such bodies of intervening space that serve to augment material of various biologically relevant sorts in such a way as to furnish a medium for life. This is because all molecular motion presupposes the existence of intervening space, since all molecules, when they move (for example when air molecules are dislodged by the physical mass of an organism), must have some portion of empty space into which they move. To use an example discussed already by Lucretius,

[when two bodies] suddenly spring apart from contact on a broad surface, all the intervening space must be void until it is occupied by air. However quickly the air rushes in all around, the entire space cannot be filled instantaneously. The air must occupy one spot after another until it has taken possession of the whole space. (De rerum natura, I, 385-397)

Every medium, then, is a mixed medium involving at least some intervening empty space. A medium is constituted out of space and matter in such a way that the tenant may move freely within it. And we shall suppose that the same medium may be constituted out of different matter at different times; as with material bodies, so too here: there may be a turnover of particles (in this case, particles of air or water).

That every medium involves some intervening empty space is important also for the proper understanding of the topological structure of niches. The medium of a niche, we want to say, fills up the environing hole and 
must therefore be in contact with the retainer. But due to the density of space, two entities can never abut each other if both are topologically closed, i.e., contain their own physical boundaries as parts. They may be so close to each other that they appear to be in contact to the naked eye, but they cannot be truly adjacent. Accordingly, the medium cannot consist exclusively of air or water molecules, or of molecules of any sort, because any such aggregate would involve a physical boundary. It would therefore qualify as topologically closed and this would imply that it could not be in genuine contact with the retainer-itself a topologically closed entity. (In this sense, Lucretius's words are misleading because they suggest that it is air molecules-rather than air as a mixed medium-that "takes possession of the whole space".) Empty space, on the other hand, contains no physical boundaries or discontinuities [27]. A body of empty space is thus never, topologically speaking, a closed entity, and it is precisely this property of empty space that allows genuine contact between the medium - the air or water molecules plus the intervening space-and its closed retainer. ${ }^{9}$

Incidentally, the principle that two closed entities can never abut (touch) each other also explains why there cannot be niches without a medium, a perfect plug (tenant) in a perfect hole (niche). This follows not only from the density of the continuum but also from standard physics, which postulates at every surface of apparent contact between two solid bodies a compaction and an exchange of molecules across the boundary but no genuine touching.

\section{Retainers and Fiat Boundaries}

Given any niche token, we can now conclude, it is the corresponding mixed medium that is at any given time the carrier of the properties represented in the corresponding niche type. The job of the retainer is to protect

\footnotetext{
${ }^{9}$ Of course, we know that at the molecular level macroscopic physical objects, too, are not continuous and do not have continuous boundaries of the sort conceived by common sense. Thus not only the medium of a niche but also its retainer and tenant are constituted by mixed stuff involving a certain amount of empty space. Consideration of these matters will be required if we are to provide a full story at the microscopic level of the relations between given types of organisms and the corresponding types of niche.
} 
the medium and to ensure that it satisfies those properties. The retainer of a niche is thus analogous to the skin or hide of an organism. It functions causally. It blocks or channels different types of causal flows and thereby brings it about that a certain niche type is instantiated in a certain region of space.

The very geometry of the retainer-medium-tenant configuration is determined by this causal function. The medium surrounds the tenant, and the retainer contributes a physical demarcation between the medium and the outer world. The retainer faces in towards the medium of the niche and thus also, in principle, towards its tenant. The theory of retainers may therefore be seen as part of the theory of surface layout in J. J. Gibson's sense [9]: retainers are affordances. In addition, the causal function of retainers requires that they enjoy a certain qualitative density which is higher than that which is manifested by the pertinent medium and tenant, and by potential intruders. A retainer must be the boundary of a solid physical structure. ${ }^{10}$ And the process of constructing niches (a process which overlaps significantly with life itself) is in no small part a process of surrounding space via the construction of suitable retainers. A given region of space becomes set aside, its contents - in the form of a medium for life-become subject to control.

But now let us consider once again the niche depicted in Figure 1. Inspection reveals that the environing hole in fact has two sorts of boundary: a solid physical boundary, corresponding to the retainer, on the one hand; and what we have elsewhere [32] called a fiat boundary (illustrated by the broken line in Figure 3), on the other. The latter is a boundary which corresponds to no physical discontinuity in the underlying material of the niche in question and to no qualitative heterogeneity in its surroundings. Fiat boundaries of this sort are similar to those which delineate connected body parts (such as your hand and your arm) or geographic features (such as bays within their surrounding seas or seas within surrounding oceans) [28]. They supplement an associated physical retainer (in our present case: the inner walls of the cave) in such a way as to form a complete boundary that fully envelopes the interior (here: the medium).

${ }^{10}$ An invisible electronic fence within which dogs run free is a limit case of a retainer by these lights. The fence is experienced by the dogs as having a physical density which it does not in fact possess. 


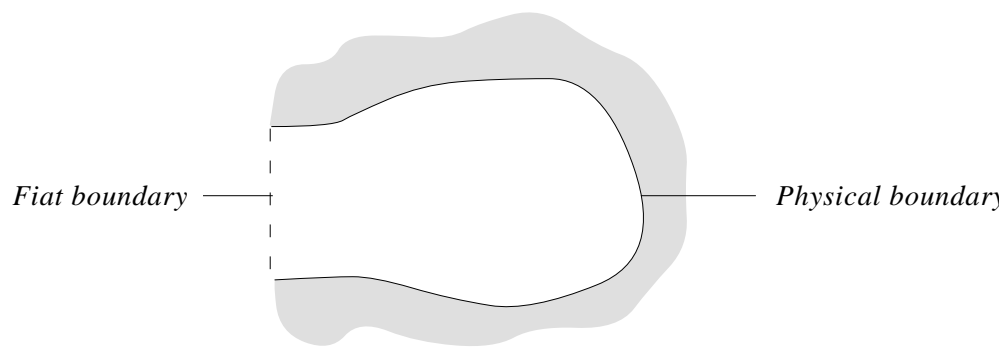

Figure 3. Two types of boundary.

Most developed niches (a cave, a shell, a house, a nest) are of this kind - they are bound partly by a physical retaining boundary and partly by a fiat boundary of this sort. This is because in ordinary circumstances an organism is free to leave its niche, for instance for hunting purposes. When, however, the bear leaves its cave, or the squirrel leaves its dray, then it enters a new niche whose retainer is a much more heterogeneous physical structure involving the surfaces of the relevant trees, leaves, meadows, rivers, ice floes, and so forth. Such niches are more ephemeral because a greater portion of their boundary is of the fiat sort. Are there also niches whose boundary is entirely of the fiat sort?

Consider, for example, a skylark flying high in the sky, or an open ocean fish. Here, too, in certain circumstances we may speak of the bird or the fish as being in a niche, a niche whose medium exemplifies the environmental properties represented in the relevant Hutchinsonian niche hypervolume. And here, too, we can recognize a double hole structure. In such cases, however, the environing hole seems not to be determined or supplemented by solid physical boundaries at all, for there is no relevant solid retaining object and no associated channeling of causal flows. Rather, we should think of the boundary around the medium of the bird or of the fish as a fiat boundary delineating a bubble-like zone or volume of space in which, at any given time, the bird or fish is housed.

The fiat boundaries in question here (whether or not they are supplemented by physical retainers) may recall borders found in the artefactual world of census tracts and air traffic corridors. The latter arise through deliberate cognitive acts of a sort which yield a direct delineation of a region or volume of space. The fiat boundaries in question here, in contrast, are indi- 
rect by-products of the behavior patterns of the organisms involved. This means, inter alia, that they may be affected to an especially high degree by the phenomenon of vagueness. While it may be determinately true of certain regions that they fall within the interior of the niche for a given bird, and determinately true of certain other regions that they fall within the exterior of this niche, there will standardly be no sharp line which constitutes a single (fiat) boundary of the niche in question. Rather, it may be that we have to deal with families of nested regions which form dense concentric clusters, each of which might at any given time qualify (perhaps to variable degrees) as the location of the relevant token niche. ${ }^{11}$

Matters are complicated still further by the phenomenon of movement from place to place. Consider a bird in flight. The bird may be viewed as inhabiting in succession a continuum of bubble-like niches, each one of which is entirely demarcated by boundaries of the fiat sort. Organisms need not, however, be in a niche at every moment of their existence. Rather, they may be en route from one niche to another. A bird might be fleeing its niche, or looking for a better niche. Or it might be migrating. A salmon swimming upwards in a waterfall is not in a niche; rather it is striving to locate and to move into a new (seasonal) niche. Thus to deal with such cases we may need not a theory of continuous niche transitions, but rather an account of what is involved when an organism leaves or enters a niche of the more familiar, static sort.

Organisms of different types and at different stages of their development will embrace different strategies in regard to the demands of security and freedom of movement in their interaction with the outside world. Common plants, for example, enjoy a maximum of protection in the earliest postgermination phases of their existence (when they are underground) and a minimum thereafter, combined with a negligible freedom of (self-moved) movement at every stage. Snails build their own protection in the form of a shell around themselves. And barnacles gain further protection by bonding

\footnotetext{
${ }^{11}$ A clustering of this sort is characteristic of many common sorts of fiat boundaries. Consider again the boundary between your hand and your arm, or between Mont Everest and the surrounding territory $[30,39]$. In such cases there is no one way to draw the line separating the one object from the other-which is why we may want to speak (loosely) of these objects as having vague boundaries.
} 
themselves to a solid surface, thereby sacrificing freedom of movement almost entirely. At the opposite end of this spectrum of alternatives is the strategy which gives rise to the purely fiat boundaries of certain niches, as in the case of the hovering bird or the open ocean fish. Here the tenant enjoys maximal freedom of movement, but this gain is combined with zero protection derived from the isolating power of solid niche walls.

A compromise between protection and freedom of movement in this sense is effected by every organism in relation to the passage of material across its own exterior boundary (its skin or hide). Compromises of this sort are necessary for the survival of every tenant, and as Ingarden puts it,

if [an] open system is to be able to sustain itself effectively within the real world for a time, as something identically the same, then it should not be universally open but must, at least in some respects, be bounded off from the surrounding world and partially isolated or, better, shielded from it. [15: 86]

It is in this sense that the job of protection and causal channeling performed by the boundaries of a niche extends the job performed by boundaries of various types on the side of the tenant itself.

\section{The Structure of Retaining}

The history of evolution is in part a history of the passage from organisms with very simple (often to a significant degree fiat) niches, which arise automatically in virtue of the actions of the organisms in question, to organisms with complex, physically walled niches which reflect the hard work of construction of retainers of appropriate types. Only niches of the latter sort have the advantage that they can survive for longer or shorter periods even in the absence of a tenant. Moreover, their greater survival-capacity, sometimes extending across generations, can justify the investment of ever greater quantities of energy and resources for the purposes of niche embellishment.

Consider again the armchair in which you are currently lodged. The niche which you are occupying is roughly bubble-shaped and it is suspended across the seat and back of the chair and up and around your body. It has a combination of fiat and physical boundaries, the former determined by the chair itself, the latter by the volume above and in front of the chair, whose shape and size are determined by your position and by your 
activities and concerns of the moment. Your current niche thus represents a compromise between physical protection and freedom of movement. This suggests that we classify niches according to the type of their exterior boundary and thus according to the degree to which their media are bound by retainers. We distinguish four main classes, corresponding to the patterns in Figure 4.
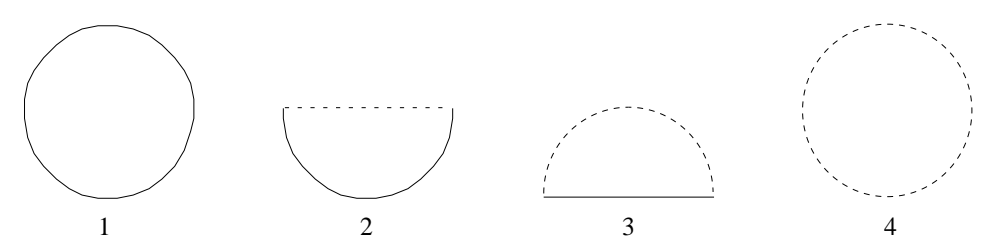

Figure 4. The four basic niche classes (cross-sections). A solid line indicates a physical retainer; dotted lines indicate fiat boundaries.

1. At one extreme we find niches that are fully bounded by a retainer. A niche of this class is an ideal niche from the perspective of protection-a more or less perfect cavity, physically protected in each direction and in relation to each pertinent family of intruders or impurities. Examples are: an egg, a closed oyster shell, the larvae chamber inside a wood-ant nest, a submarine, a car, a nuclear clean room facility, an incubating tent. ${ }^{12}$ We may include here also niches such as a fine-mesh cage, whose surrounding physical structure, though incomplete in some degree, is sufficiently dense to supply a protective retainer which fully circumscribes the relevant niche. Class 1 niches can be subdivided further into those whose retainers offer full protection without access points (a full passive defense, such as that provided by a larval cocoon) and those with defended access points (such as an oyster shell, which must actively keep its shell closed to prevent predators from prying it open). The degree of protection, in turn, will depend on the physical properties of the retainer: the walls of the crocodile's egg, for example, have a higher protective value than the thin membrane which is the zona pellucida of the mammalian zygote.

\footnotetext{
${ }^{12}$ Perhaps also: a womb. On the implications of our present deliberations for the understanding of the relations between an embryo or fetus and its uterine environment, see [29].
} 
2. Most niches are, like the bear's cave, not fully bounded, but rather bounded only to a certain degree. Examples are: a kangaroo-pouch, a nest, a hive, a cabriolet. All of these are niches which, geometrically, do not involve closed cavities but rather natural or artificial hollows within their respective environments, with a fiat boundary marking (more or less vaguely) the opening. Nevertheless they are for a range of different reasons fairly robust from the perspective of protection. Trenches (in battlefields) are of this kind. We may further include in this category niches with multiple openings-tunnel niches - such as a person's stomach (which can be a niche for a parasite), or a path cleared in the jungle (which can be a niche for hunters).

3. Some niches are bounded by a partial retainer which offers no or a very low degree of protection - by a floor, for example, or by a single wall. This is the case of the niche around two people chatting on the sidewalk (bounded by the pavement beneath them) or the niche of the oxpecker removing ticks from the back of an African rhinoceros (bounded by a part of the rhinoceros's hide). We may include in this category also niches which are bounded on two sides: consider a pedestrian with an umbrella.

4. Finally, at the other extreme of the continuum between bound and free, we find niches that lack a retainer altogether. Such niches are just bubble-like zones, as in the case of the niche of the fish orbiting underwater. This class of niches, too, may manifest a range of different topologies. When a falcon is flying in the sky circling above the area where its prey is to be found, the niche of the falcon is its orbit, a torus-shaped region that is bordered, again, by boundaries of the fiat sort.

As will be clear from the examples considered, our classification is a simplification of the spectrum of cases actually realized in the natural world. When fully systematized, the full spectrum determined by the geometry of the retainer-fiat boundary structure might be counted as an additional axis in the array of niche types defined under the hypervolume conception.

Our classification is simplified not least in that it does not as yet do justice to the ways in which the physical boundaries of niches-particularly of higher organisms - may involve mixed retainers, which is to say retainers blocking or channeling causal flows of different types. Thus for example both walls and windows may serve as parts of a single retainer. Often, a niche of class 2 can be transformed into a niche of class 1 by adding a plug- 
or door-like structure or by augmenting the surroundings in such a way as to replace all fiat boundaries by boundaries of the physical sort. More generally, one can move along the continuum by adding or removing portions of the relevant retainer. Thus, niches of classes 2 and 3 are topologically equivalent, but they differ from the perspective of protection. As we saw, the freer the niche, the easier it is for the tenant to move out of it (for example when fleeing from predators). Finally, there are niches of type 4 which, though lacking a retainer altogether, are marked by boundaries that are not entirely of the fiat sort. When an octopus secretes black ink to protect itself, the ink determines a physical boundary between the medium and the exterior of the niche which serves to simulate a genuine retainer. Similarly, consider the fish in a thermocline, a beach-dweller in the shade, or the planet earth in its atmosphere. Cases such as these are of type 4 . But even though the relevant boundaries are not retainers on our conception, the niches in question are still marked out physically from their surrounding environments. By taking such cases into account, the theory here presented can serve as a starting point for a general theory of causally relevant spatial volumes.

Note, further, that some organisms may occupy niches that involve combinations of the protective features distinguished above. Plants, for example, have a double medium: above the ground their niche structure is of class 3; below the ground the structure is of class 2 . The different parts of a single tropical rain forest vine may simultaneously occupy a multiplicity of niches of different types, these different parts manifesting correspondingly different qualities of leaf shape, leaf spacing, phototropism and geotropism depending on the incident light conditions.

We may further subclassify niches of classes 1 through 3 into those which are stationary (a larval chamber; a rabbit hole; a meadow) and those which are mobile (a womb; a snail's shell; the back of a rhinoceros). It seems however that class 4 niches can only be stationary: in the absence of a retainer there is no mechanism whereby the medium would follow the tenant when the latter moves from place to place. ${ }^{13}$ (The niche is not as it were dragged along with the tenant.)

${ }^{13}$ All of this is of course to be understood modulo relativity theory, the movement of the planet, etc. In fact, when we talk of regions of space we are talking of regions somehow bonded or localized in relation to the surface of the earth. 


\section{Niche Construction}

Let us finally look at the different ways in which an organism may come to occupy or possess a niche and to interact with the niches of other organisms.

We may distinguish two basic ways in which the organism may become a niche tenant. In the first case, the organism enters a pre-existing vacant niche: the bear occupies a cave and evicts its prior tenant. In the second case there is no pre-existing niche: a beaver builds a beaver dam; a worm creates a wormhole; a falcon pair creates a plucking site; an embryo creates a cavity within the uterine wall. As these examples make clear, niches of many different types arise in symbiosis with the activities of organisms or groups of organisms; they are not already there, like vacant rooms in a gigantic evolutionary hotel, awaiting organisms who would evolve into them. In this sense, as Lewontin puts it,

organisms not only determine what aspects of the outside world are relevant to them by peculiarities of their shape and metabolism, but they actively construct, in the literal sense of the word, a world around themselves. [22: 54] ${ }^{14}$

This is especially true in the case of niches of classes 1 and 2, that is, niches with a physically protecting retainer. In such cases, the building of a niche (what some call "ecosystem engineering" [17]) is a complex process that typically involves activities and metabolic processes through which an organism or population modifies the environment. This process involves changing properties of the surrounding environment as for example when animals build houses and nests. They may then work hard to improve or repair their niches, in relation both to media (as when you turn up the heating in your tent) and retainers (as when you mend the roof of your house). We can in this light distinguish intuitively between felicitous niches, within which organisms flourish, and critical niches, within which organisms fail to flourish, sometimes catastrophically. A token niche is felicitous if, for every di-

\footnotetext{
${ }^{14}$ Lewontin himself appears to rely on both niche type and niche token concepts here, for only concrete niches, one presumes, can be literally constructed. In fact, as Robert Brandon [1] has emphasized, the idea that the external world or environment is to a significant extent constructed by the organism has different meanings depending on the relevant notion of 'environment'. (See also [35], ch. 11.)
} 
mension of the relevant hypervolume (niche type), the relevant variables are within the threshold values; otherwise it is critical.

Constructing a niche typically involves the building of artefacts, as when a bird builds a nest or people build houses. In many cases, however, the niche is not an artefact-thus it is not a new, positive object in its own right, but rather merely the modification of a pre-existing habitat, as when a worm creates a wormhole or an insect chooses a water-filled cavity as site for oviposition [16]. The case of plants is somewhat intermediate between these two. The niche of a plant is the result of a continuous process which changes relevant environmental factors such as exposure to sunlight and the chemical composition (humidity, salinity, acidity, etc.) of the soil, while at the same time taking advantage of the solid and enduring retaining structure which this soil provides $[6,12]$.

Processes of this sort can be expressed also in terms of the Hutchinsonian conception. Thus suppose that presence of a pond is a dimension of the hyperspace-niche of some species of vertebrate X (say: beavers). Suppose further that some given region $\mathrm{Y}$ satisfies all the other critical values of the hyperspace-niche for $\mathrm{X}$ but does not have a pond. Now however the beaver, by constructing a dam, produces a pond which moves region Y's position in the hyperspace from presence of a pond $=$ FALSE to presence of $a$ pond $=$ TRUE.

The theory of the construction and maintenance of niches is important also for evolutionary theory. Recent work [19] suggests that niche construction may result in selective processes that outweigh other sources of selection, sometimes to the point of generating novel or unusual evolutionary outcomes. Some ant and termite species, for instance, have developed the habit of plugging the entrances to their nests at night in order to regulate temperature. It has also been argued that niche construction may influence the genetic variation in a population. Adaptation, under such conditions,

ceases to be a one-way process, exclusively a response to environmentally imposed problems: instead it becomes a two-way process, with populations of organisms setting as well as solving problems. Evolution consists of mutual and simultaneous processes of natural selection and niche construction. [20: $§ 1.1]$

\section{Niche Interaction}

Different types of niche interaction can now be distinguished, reflecting dif- 
ferent types of interaction on the part of the relevant tenants. In particular, we may draw a distinction based on whether or not the interaction is between niches on different trophic levels, i.e., different levels of the food chain (or of what more properly should be called the food web, in view of the fact that organisms typically consume more than one type of animal or plant and thus inhabit niches that are tied by dependence to more than one type of underlying niche within the hierarchy).

The biologically most important family of niche interactions are of the first type, i.e., between niches on different trophic levels. Four sorts of links in the chain or web can be distinguished:

1. at the bottom of the hierarchy is the saprophytic chain, in which micro-organisms live on dead matter;

2. above this is the primary relation between animals and the plants they consume;

3. above this is the predator chain, in which animals of one sort eat smaller animals of another sort;

4. crosscutting all of these is the parasite chain, in which a smaller organism consumes part of a larger host organism.

If, now, a higher niche rests on or involves lower organisms, then the question arises whether these lower organisms are to be conceived as parts of the retainer of this higher niche, as intruders within its medium, or as parts of this medium. For lowest-level organisms it is tempting to regard the floor of dead matter over which they crawl as the retainer of their niche. Such a view is less tempting, however, in the case of the predator and its prey.

Consider now the case where the interaction is between niches on the same trophic level, as when a dominant male within a colony is challenged by a younger aggressor, or when male and female conspecifics engage in mating rituals. Suppose a cat approaches the lair where another cat is resting. We may then distinguish a range of cases of two-tenant, two-niche interaction of this type (Figure 5).

Case a: The two cats are friendlily disposed towards each other. Their respective niches may then fuse, creating a new niche with a new, two-cat tenant.

Case b: The two cats are strangers. Each retains its own niche and the two niches may come to overlap. The intruding cat may itself come to over- 
lap (that is to say: enter) the niche of the stationary cat; this is a mark of the fact that we are here dealing with fiat boundaries. Alternatively, the two niches may merge and the situation may again resolve itself into one in which a joint niche is created.

Cases $\mathrm{c}$ and $\mathrm{d}$ : The intruding cat is in fact an enemy, who will attempt to overpower the resting cat. In this case neither the intruder nor its niche will overlap with the niche of the intrudee. Rather, the intrusion will cause in the latter a deformation, which will be symmetrical or asymmetrical according to the relative strengths of the animals involved. (More complex patterns of interaction, with one niche partly or completely surrounding the other, are possible too. Think of a falcon approaching a bird hovering in the sky and starting to orbit around it.)
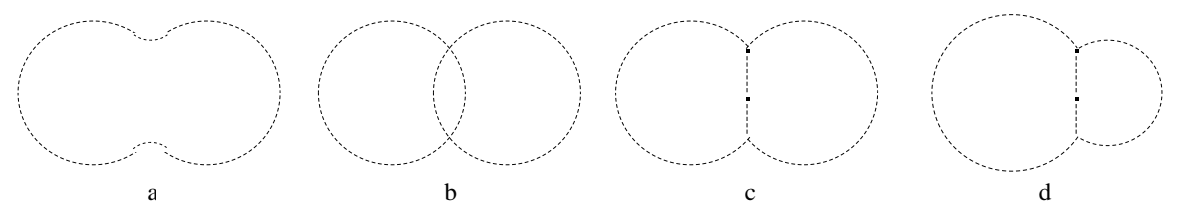

Figure 5. Niche interaction patterns: fusion (a); overlap (b); symmetric deformation (c); asymmetric deformation (d).

These ideas can serve as a basis for a theory of the ways in which population interactions are projected into the spatial dimension. In conditions of low population density, birds of a single species will establish cylindrical territories for themselves which have roughly circular cross-sections and which are separated from their neighbors' territories by no-bird's zones of undefended space. As population increases an exhaustive tiling is effected, analogous to the tiling of a beehive, which divides the whole space into roughly hexagonal areas [38].

These patterns of interaction are common not only in the realm of ecology in the narrow biological sense but also in relation to broader phenomena of territoriality. The studies of Edward T. Hall [11] and others on personal space, for example, reveal how each person uses verbal and nonverbal clues to establish a surrounding space, a space which has different dimensions and different degrees and types of elasticity in different cultures. This personal space or fiat territory around your body is carried around with 
you when you move. Each of the concentric bubble-like spaces distinguished by Hall satisfies the conditions for being a (token) niche in our sense. And it is tempting to postulate that every organism, at least every higher organism in a waking state, is always in a niche of at least this sort-a (vaguely defined) niche of class 3 or 4 .

\section{Beyond Biology}

We referred above to a spectrum of alternatives in the organic world regarding safety vs. freedom of movement, and we pointed to the role played by physical and fiat boundaries of niches in the realization of these alternatives. It is now clear that a similar spectrum of alternatives is present also in the realm of human artefacts, ranging from underground nuclear shelters and soccer stadiums to heliports and bodies of controlled air space around airports. Our taxonomy of tenant-niche structures may be applied to such cases also. And once again, we can often distinguish hierarchies of nested niches.

When considering the constructed, physical niches of complex organisms such as ourselves we often find a series of nested retainers stacked concentrically inside each other. Thus, for example, you may be inside a sleeping bag inside a cubicle (a hollow niche), inside an office, which has windows (creating a closed but light-permeable niche retainer), facing out onto a garden (another hollow niche, bounded by trees and fences), which is itself part of a park-like structure surrounded by a wall. Each of these different surrounding levels involves a different sort of control of its immediate surrounding medium (for example: different sorts of climate and noise control). In addition they will involve different sorts of control over the admission and exclusion of other organisms - an analogue, on the level of niches, of the workings of the immune system on the level of individual organisms.

The niche of the driver of a car is the interior of the car, the niche of the car itself is the road along which the car is driving. The niche of the astronaut is the interior of her spacesuit, the niche of the astronaut-plusspacesuit is the interior of the spaceship, the niche of the spaceship is the relevant region of space. Note that such a nesting of niches does not constitute a counterexample to the thesis (defended in [31]) to the effect that tenants and niches are categorially disjoint. The spaceship might at first sight 
seem to play both roles-it is a niche (of class 1) and it has a niche (of class 4) - but really it is a different entity that is involved in the two cases: on the one hand the spaceship interior (including the interior surfaces of its walls); on the other hand the whole spaceship (including the exterior walls).

These examples illustrate how the niche concept which we have been developing may find application within domains that go beyond the concerns of biology and ecology. Let us then conclude by considering three last groups of examples, ordered in terms of increasing abstractness.

1. Niches and Territoriality. Anthropologists have shown that the force of territoriality diminishes with increase in group size and in spatial area, and that, in the case of both human and non-human animal species, a nested continuum of types of site must be distinguished: from a large home range (that area within which the group spends most of its time, including foraging and hunting) to narrow territories (in relation to which the occupying individual or group actually demands exclusive use) [36]. Most anthropologists today would argue that territoriality in the narrow, biological sense applies only to small (roughly: family-sized) groups. As far as application to larger groups is concerned, they prefer to speak instead of the much weaker and more variegated phenomenon of territorial functioning, defined as

an interlocked system of sentiments, cognitions, and behaviors that are highly place specific, socially and culturally determined and maintaining, and that represent a class of person-place transactions concerned with issues of setting management, maintenance, legibility, and expressiveness. [36: 6]

Alternatively, they talk of territoriality not in terms of defense and exclusive use but rather in terms of

the attempt by an individual or group to affect, influence, or control people, phenomena, and relationships, by delimiting and asserting control over a geographic area [the territory]. [26: 19]

Examining the history of the very earliest human settlements we encounter the following patterns. Groups exhibit a tendency to expand into ever larger regions until they reach physical obstacles such as coastlines, or until they meet the resistance of an equal and opposite expansion on the part of neighboring groups. Mutual adjustments very similar to those effected 
spontaneously by bubbles on the surface of a soapy solution are then encountered, and what look like clusters of bubbles can indeed be found on maps depicting tribal expansion in early Africa. As in the case of bird territories in a region subject to pressure of population (and as in the case of the physical tiling of a beehive), so also in the geographic case we can witness a tendency towards (fiat) tilings into broadly hexagonal units.

Various patterns of territorial evolution can then be established, including the various patterns of war and conquest, where a group occupying a given territory is evicted or destroyed by a second group. Expansion can first of all lead to the peaceful merging of groups (through intermarriage and through trade and other forms of cooperation) leading also to a concomitant merging of the underlying territories. Expansion and competition for territory can lead to an absorption by one dominant group of smaller or less powerful groups and it may provoke also a splitting off of subgroups who proceed to establish new, disconnected territories in other regions.

2. Niches as Power Projection Zones. Imagine a region in the ocean which is occupied by two ships of opposing fleets. Each ship, we can say, has its own niche, which can be defined in terms of its effective power projection zone: the boundary of the niche is determined by the distance across which the ship can effectively defend itself against an enemy. What happens when the two ships approach each other? The niche interaction patterns considered above in relation to biological niches apply naturally in this case also. It is tempting to say that the two niches come to overlap as in Figure 5b; but this would ignore the defensive capability that is built into the very notion of effective projection of power. Rather, the correct pattern of interaction is that of Figure 5c: the two niches come into contact and press against each other, and each party is trying to breach the stand-off by breaking into its opponent's niche (Figure 5d).

Considerations like those embraced in our niche ontology are indeed implemented in maritime technology for information management and peacetime collision avoidance [24], for example in automatic radar plotting aids (ARPA). Many systems of this sort employ the concepts of collision and guard zones. A guard zone is a zone of some selected geometry around a ship that the captain chooses to implement on the ARPA. The default guard 
zone is elliptical, with the ship off-center because there is need for a larger guard zone in front of the ship in the direction of movement. The function of a guard zone is to give a warning signal when a target (for example another ship) crosses this boundary, at which time it will also be automatically plotted by the system (it will be given a vector indicating speed and direction). When a ship has crossed the guard zone the captain can choose to set up another zone, this time around the plotted target ship, called the collision zone. This zone will follow the plotted ship dynamically as it is tracked on the ARPA and thus enable the ship's systems to adjust course automatically in such a way as to avoid collision.

There are similar applications in the area of air traffic control automation. The "volume of protected airspace" enclosing an aircraft-a notion that is central to air traffic alert and collision avoidance systems-is in many respects like the niche of a hovering bird [7].

3. Niches as containers. The niche theory outlined above can be applied also at a different level of abstraction, for instance to provide an account of certain semantic phenomena. Consider the range of different meanings of 'in' captured in:

the fruit is in the bowl

the bird is in the nest

the lion is in the cage

the pencil is in the cup

the finger is in the thimble

the fish is in the river

the river is in the valley

the water is in the lake

the car is in the garage

the fetus is in the cavity in the uterine lining

the colony of whooping crane is in its breeding grounds

All these uses of 'in' are spatial, but the situations depicted in the different cases cannot be captured by any simple geometrical reading of 'in'. Even geometry plus topology will allow us to do justice to only some of the distinctions involved [37]. The theory of niches, by contrast, appears to provide a general and overarching framework within which all these distinct uses 
can be captured in a natural way. The same framework can account also for the referents of a range of everyday terms — such as 'valley', 'hole', 'home', 'room', 'café', 'building', 'place', 'neighbourhood'-in a way which is more adequate than standard lexical semantic accounts (in terms of objects and regions of space). When we say 'Get into the car', for example, we mean something quite specific. We do not mean 'Climb into the trunk', or 'Hide under the hood'. We are thinking of the car as a niche and we are asking you to enter it as a tenant enters its niche. ${ }^{15}$

\section{Concluding Remarks}

Biological selection theory is concerned with phenomena at the level of populations. Hence, as Sober and Lewontin have pointed out, it is 'concerned with what properties are selected for and against in a population. We do not describe single organisms and their physical constituents one by one.' [34: 172] Selection theory, in other words, is about genotypes. But if there are genotypes, then there are also genotokens; and just as there are genotokens so also, we submit, there are phenotokens, and then also niche tokens, and we can now reiterate that it is the latter, above all, which are the object of the present essay.

The hypervolume theory of the niche as used by ecological scientists is a theory of niche types. Biologists and ecologists are interested in averages, in statistical laws and regularities; it is on the level of regularities that adaptation, which is a matter of relations between organism types and environment types, arises. But consider such general laws as Grinnell's competitive exclusion principle [10], which states that, in competition between species that seek 'the same ecological niche', one species survives while the other expires under a given set of environmental conditions. Such laws can be applied if, and only if, both niche types and niche tokens are acknowledged within the same domain of application.

The theory outlined above is designed, therefore, as a contribution to the philosophical foundations of biological science. The theory is not concerned with the task of formulating laws or regularities of a biological sort,

\footnotetext{
${ }^{15}$ In this sense, the role of niches in spatial representation generalizes the role of holes: see [3], ch. 8 .
} 
for example via averaging or other quantitative techniques. Rather, the theory deals in general principles to the effect that, for example, there is no niche without a medium, there is no medium without a tenant, and so on. Thus it deals, also, in those aspects of the organism-environment fitting relation which are so fundamental (or so trivial) that biological science has tended to ignore them. Types can exist only through their corresponding tokens. Our theory of token niches is meant to plug this gap in the foundations of biology, and to illustrate its ramifications beyond the sphere of biology narrowly conceived and out into the realms of culture and technology.

\section{Acknowledgements}

This material is based upon work supported by the American Philosophical Society and by the National Science Foundation under Grant No. BCS-9975557, a preliminary report of which appeared as [33]. Thanks to Robert Brandon, Berit Brogaard, Roberto Casati, Mike Happold, David Mark, and Michael May for helpful comments.

\section{References}

1. Brandon, R. N., 1990, Adaptation and Environment, Princeton, NJ: Princeton University Press.

2. Casati, R., and Varzi, A. C., 1994, Holes and Other Superficialities, Cambridge, MA, and London: MIT Press.

3. Casati, R., and Varzi, A. C., 1999, Parts and Places: The Structures of Spatial Representation, Cambridge, MA, and London: MIT Press.

4. Damuth, J., 1985, 'Selection among "Species": A Formulation in Terms of Natural Functional Units', Evolution 39, 1132-1146.

5. Eldredge, N., 1989, Macroevolutionary Dynamics: Species, Niches, and Adaptive Peaks, New York: McGraw-Hill.

6. Ellis, S., and Mellor, A., 1995, Soils and Environment, London: Routledge.

7. Ford, R. L., 1986. 'The Protected Volume of Airspace Generated by an Airborne Collision Avoidance System', Journal of Navigation 39, 139-158.

8. Freeman, J., and Hannan, M. T., 1983, 'Niche Width and the Dynamics of Organizational Populations', American Journal of Sociology 88, 1116-1145.

9. Gibson, J. J., 1979, The Ecological Approach to Visual Perception, Boston: Houghton-Mifflin.

10. Grinnell, J., 1917, 'The Niche-Relationships of the California Thrasher', The Auk 34: 427-433.

11. Hall, E. T., 1966, The Hidden Dimension, Garden City: Doubleday.

12. Holmgren, M., Scheffer, M., and Huston, M.A., 1997, 'The Interplay of Facilitation and Competition in Plant Communities', Ecology 78: 1966-1975. 
13. Hurlbert, S. H., 1981, 'A Gentle Depilation of the Niche, Dicean Resource Sets in Resource Hyperspace,' Evolutionary Theory 5: 177-184.

14. Hutchinson, G. E., 1978, An Introduction to Population Ecology, New Haven: Yale University Press.

15. Ingarden, R., 1984, Man and Value, Munich: Philosophia Verlag.

16. Jaenike, J., 1982, 'Environmental Modification of Oviposition Behaviour in Drosophila', American Naturalist 119: 784-802.

17. Jones, C. G., Lawton, J. H., and Shachak, M., 1997, 'Positive and Negative Effects of Organisms as Physical Ecosystem Engineers', Ecology 78: 1946-1957.

18. Lack, D., 1974, Evolution Illustrated by Waterfowl, London: Blackwell.

19. Laland, K. N., Odling-Smee, F. J., and Feldman, M. W., 1996, 'On the Evolutionary Consequences of Niche Construction', Journal of Evolutionary Biology 9: 293-316.

20. Laland, K. N., Odling-Smee, J., and Feldman, M. W., 2000, 'Niche Construction, Biological Evolution and Cultural Change', Behavioral and Brain Sciences 23, 131-146.

21. Lewontin, R., 1979, 'Sociobiology as an Adaptationist Program', Behavioral Science 24, 5-14.

22. Lewontin, R., 2000, The Triple Helix: Gene, Organism and Environment, Cambridge (MA): Harvard University Press.

23. Looijen, R. C., 1995, 'On the Distinction Between Habitat and Niche, and Some Implications for Species' Differentiation', in T. A. F. Kuipers and A. R. Mackor (eds.), Cognitive Patterns in Science and Common Sense, Amsterdam and Atlanta: Rodopi, pp. 87-108.

24. Malyankar, R., 1999, 'Creating a Navigation Ontology', Workshop on Ontology Management (AAAI-99, Orlando, FL), Tech. Rep. WS-99-13, Menlo Park: AAAI Press, pp. 48-53.

25. Odum, E. P., 1971, Fundamentals of Ecology, 3rd Edition, Philadelphia: Saunders.

26. Sack, R. D., 1986, Human Territoriality. Its Theory and History, Cambridge: Cambridge University Press.

27. Smith, B., 1995, 'More Things in Heaven and Earth', Grazer Philosophische Studien 50, 187-201.

28. Smith, B., 2001, 'Fiat Objects', Topoi 20, 131-148.

29. Smith, B., and Brogaard, B., 2002, 'Sixteen Days', forthcoming in Journal of Medicine and Philosophy.

30. Smith, B., and Mark, D., 2002, 'Do Mountains Exist?', forthcoming in Environment and Planning B (Planning and Design).

31. Smith, B., and Varzi, A. C., 1999, 'The Niche', Noûs 33, 214-238.

32. Smith, B., and Varzi, A. C., 2000, 'Fiat and Bona Fide Boundaries', Philosophy and Phenomenological Research 60, 401-420.

33. Smith, B., and Varzi, A. C., 2001, 'Environmental Metaphysics', in U. Meixner (ed.), Metaphysics in the Post-Metaphysical Age, Vienna: öbv\&hpt, pp. 231-239

34. Sober, E., and Lewontin, R., 1982, 'Artefact, Cause and Genic Selection', Philosophy of Science 49, 157-180.

35. Sterelny, K., and Griffiths, P., 1999, Sex and Death: An Introduction to the Philosophy of Biology, Chicago: University of Chicago Press. 
36. Taylor, R. B., 1988, Human Territorial Functioning. An Empirical, Evolutionary Perspective on Individual and Small Group Territorial Cognitions, Behaviors and Consequences, Cambridge: Cambridge University Press.

37. Vandeloise, C., 1994, 'Methodology and Analyses of the Preposition in', Cognitive Linguistics 5, 157-84.

38. van Riper, C. III, 1984, 'The Influence of Nectar Resources on Nesting Success and Movement Patterns of the Common Amikihi (Hemignathus virens)', The Auk 101, $38-46$.

39. Varzi, A. C., 2001, 'Vagueness in Geography', Philosophy and Geography 4: 49-65. 